\title{
Asymptotic behavior of non-autonomous stochastic Gilpin-Ayala predator-prey model with jumps
}

\author{
Yanhua Zhang \\ Institute of Oceanology, Chinese Academy of Sciences, Qingdao 266071, China. \\ University of Chinese Academy of Sciences, Beijing 100049, China. \\ College of Science, China University of Petroleum, Qingdao 266580, China.
}

Communicated by A. Atangana

\begin{abstract}
In this paper, a non-autonomous stochastic Gilpin-Ayala predator-prey model with jumps is studied. Firstly, we show that this model has a unique global positive solution under certain conditions. Then, we discuss the sufficient conditions for stochastically ultimate boundedness and obtain the asymptotic behavior of the solution. Finally, sufficient criteria for extinction of all prey and predator species, stochastic weak persistence in the mean of prey species are established. (C) 2017 All rights reserved.
\end{abstract}

Keywords: Gilpin-Ayala predator-prey model, jumps, moment boundedness, asymptotic behavior, extinction. 2010 MSC: 60H10, 92D25, 34F05.

\section{Introduction}

In population dynamics, predator and prey phenomena are interesting topics due to their universal existence and importance. The predator-prey model is composed of differential equations which describe predator-prey dynamics. The classical Lotka-Volterra predator-prey model which was developed independently by Lotka [17] and Volterra [24] in the 1920's is given by

$$
\dot{x}(t)=x(t)(a-b y(t)), \quad \dot{y}(t)=y(t)(-c+d x(t)),
$$

where $x(t)$ and $y(t)$ denote the prey and predator population size, respectively, at time $t . a, b, c, d$ are positive constants. $\mathrm{a}$ and $\mathrm{b}$ are the fixed growth and mortality rates of the prey component, respectively. $\mathrm{d}$ and $\mathrm{c}$ are the fixed growth and mortality rates of the predator component, respectively. This model has become one of classical models in the mathematical biology research, many models are based on it (see e.g., $[1,10])$. However, in the practical case, population systems are often subject to various stochastic small perturbations. It is therefore useful to reveal how the perturbation affects the population systems. As a matter of fact, stochastic Lotka-Volterra systems driven by Brownian motion have been studied extensively (see e.g., $[12,18,19])$.

Email address: ggxpzyh@163.com (Yanhua Zhang)

doi:10.22436/jnsa.010.04.62 
Unfortunately, in the Lotka-Volterra model, the rate of change of the size of each species is linear function of sizes of the interacting species $[7,14]$. This is almost impossible in complex ecosystem. In 1973, Gilpin and Ayala [8] have given a modification for Lotka-Volterra model, called Gilpin-Ayala model. This system more accord with the actual situations, and the Lotka-Volterra model is its special case. For various forms about the Gilpin-Ayala system readers can see [6, 11, 15] and references therein for details. Gilpin-Ayala competition models in random environments have recently also been studied by many authors, for example, [13, 14, 22, 23]. A stochastic Gilpin-Ayala predator-prey model with time delay and a special case of it have been studied by Vasilova [21]:

$$
\begin{aligned}
d x_{i}(t)=x_{i}(t)\{ & \left.r_{i}(t)-\sum_{j=1}^{d}\left(a_{i j}(t) x_{j}^{\alpha_{i j}}(t)+b_{i j}(t) x_{j}^{\beta_{i j}}\left(t-\tau_{i j}(t)\right)\right)\right] d t \\
& \left.+\sum_{j=1}^{d} \sigma_{i j}(t) x_{j}^{\theta_{i j}}(t) d w_{j}(t)\right\}, i=1, \cdots, m, \\
d x_{i}(t)=x_{i}(t)\{ & {\left[-r_{i}(t)+\sum_{j=1}^{m}\left(a_{i j}(t) x_{j}^{\alpha_{i j}}(t)+b_{i j}(t) x_{j}^{\beta_{i j}}\left(t-\tau_{i j}(t)\right)\right)-\sum_{j=m+1}^{d}\left(a_{i j}(t) x_{j}^{\alpha_{i j}}(t)\right.\right.} \\
& \left.\left.\left.+b_{i j}(t) x_{j}^{\beta_{i j}}\left(t-\tau_{i j}(t)\right)\right)\right] d t+\sum_{j=1}^{d} \sigma_{i j}(t) x_{j}^{\theta_{i j}}(t) d w_{j}(t)\right\}, i=m+1, \cdots, d,
\end{aligned}
$$

where $x_{i}(t), i=1, \cdots, m$, denote the population sizes of prey species at time $t, x_{i}(t), i=m+1, \cdots, d$, denote the population sizes of predator species at time $t, \alpha_{i j}, \beta_{i j}$ are positive constants and represent nonlinear measures of interspecific or intraspecific interference, $r_{i}(t), a_{i j}(t)$ and $b_{i j}(t)$ are continuous, bounded and nonnegative on $[0, \infty), r_{i}(t), i=1, \cdots, m$, are the intrinsic growth rates at time $t, r_{i}(t), i=$ $m+1, \cdots, d$, are the mortality rates at time $t, a_{i j}(t)$ and $b_{i j}(t)$ denote the effects of interspecific $(i \neq j)$ and intraspecific $(i=j)$ interaction at time $t$, and for $i, j=1, \cdots, d, \tau_{i j}(t)$ are nonnegative, bounded, continuously differentiable functions on $[0, \infty), \sigma_{i j}(t)$ are the intensities of noise at time $t, \sigma_{i j}(t)$ are continuous, bounded and nonnegative on $[0, \infty)$ and $\theta_{i j}$ are nonnegative constants. They discussed their properties of moment boundedness, pathwise estimation, stochastically ultimate boundedness, extinction and so on.

On the other hand, the population systems may suffer sudden environmental perturbations, that is, some jump type stochastic perturbations, e.g., earthquakes, hurricanes, epidemics and so on [4]. Stochastic integrals driven by Brownian motion can not describe these phenomena. But stochastic integrals with respect to a Poisson counting measure can explain these phenomena well, so introducing a jump process into the underlying population dynamics makes feasible. In [4,5], Bao et al. firstly investigated LotkaVoterra population dynamics with jumps, and gave some important results which revealed that jump processes can bring their effect on the properties of the systems. To the best of our knowledge, to this day, still no scholars investigate the Gilpin-Ayala predator-prey model with jumps which is more realistic than the Lotka-Voterra population dynamics.

In this paper, we investigate the general non-autonomous stochastic Gilpin-Ayala predator-prey model with $m$ prey and $(d-m)$ predator species with jumps which has the forms as follows:

$$
\begin{aligned}
d x_{i}(t)=x_{i}\left(t^{-}\right)\left\{\left[r_{i}(t)-\sum_{j=1}^{d} a_{i j}(t) x_{j}^{\alpha_{i j}}\left(t^{-}\right)\right] d t+\sigma_{i}(t) d w(t)+\int_{\mathbb{Y}} c_{i}(t, u) \widetilde{N}(d t, d u)\right\}, i=1, \cdots, m, \\
d x_{i}(t)=x_{i}\left(t^{-}\right)\left\{\left[-r_{i}(t)+\sum_{j=1}^{m} a_{i j}(t) x_{j}^{\alpha_{i j}}\left(t^{-}\right)-\sum_{j=m+1}^{d} a_{i j}(t) x_{j}^{\alpha_{i j}}\left(t^{-}\right)\right] d t+\sigma_{i}(t) d w(t)\right. \\
\left.+\int_{\mathbb{Y}} c_{i}(t, u) \widetilde{N}(d t, d u)\right\}, i=m+1, \cdots, d,
\end{aligned}
$$


here $x\left(t^{-}\right)$is the left limit of $x(t), N$ is a Poisson counting measure with characteristic measure $\lambda$ on a measurable subset $\mathbb{Y}$ of $[0, \infty)$ with $\lambda(\mathbb{Y})<\infty$, and $\widetilde{N}(d t, d u):=N(d t, d u)-\lambda(d u) d t, w(t)$ is a one dimensional standard Browian motion. We assume $w$ and $N$ are independent. $c_{i}(t, u)$ are bounded functions, $c_{i}(t, u)>-1, u \in \mathbb{Y}, i=1, \cdots, d$. Other parameters are defined as in [21].

Throughout the full text, $\left(\Omega, \mathcal{F},\{\mathcal{F}\}_{t} \geqslant 0, \mathbb{P}\right)$ is a complete probability space with a filtration $\{\mathcal{F}\}_{t} \geqslant 0$ satisfying the usual conditions. We denote $R_{+}^{d}=\left\{x \in R^{d}: x_{i}>0\right.$ for all $\left.i=1, \cdots, d\right\}$. If $x \in R^{d}$, its norm is denoted by $|x|=\left(\sum_{i=1}^{d} x_{i}^{2}\right)^{\frac{1}{2}}$. If $A$ is a matrix, its trace norm is denoted by $|A|=\sqrt{\operatorname{trace}\left(A^{\top} A\right)}$. If $f(t)$ is a continuous bounded function on $[0, \infty)$, then define

$$
f^{\mathfrak{u}}=\sup _{t \in[0, \infty)} f(t), \quad f^{l}=\inf _{t \in[0, \infty)} f(t) .
$$

For any constant sequence $\left\{c_{i j}\right\}(i, j=1, \cdots, d)$, define

$$
\left(\check{c}_{i j}\right)=\max _{1 \leqslant i, j \leqslant d} c_{i j}, \quad\left(\check{c}_{i i}\right)=\max _{1 \leqslant i \leqslant d} c_{i i}, \quad\left(\hat{c}_{i j}\right)=\min _{1 \leqslant i, j \leqslant d} c_{i j}, \quad\left(\hat{c}_{i i}\right)=\min _{1 \leqslant i \leqslant d} c_{i i} .
$$

Assumption 1.1. $\left(\hat{a}_{i \mathfrak{i}}^{l}\right)>0,\left(\hat{\alpha}_{i i}\right)>\max _{D_{1}}\left\{\alpha_{i j}\right\}$, where $D_{1}=\{(i, j): m+1 \leqslant i \leqslant d, 1 \leqslant j \leqslant m\}$.

The main aim of our work is to study the properties of non-autonomous Gilpin-Ayala $m$ prey and $(d-m)$ predator species model with jumps. The remaining part of this paper is organized as follows. In Section 2, we show that the solution of (1.1) is global and positive under certain conditions, i.e., the population will not explode in a finite time, which is logical since $x_{i}$ represents the size of the $i$-th species. Section 3 and 4 deal with the stochastically ultimate boundedness and asymptotic pathwise behavior of the solution, respectively. Finally, we carry out the survival analysis for our model and obtain some sufficient conditions for extinction of all species and stochastic weak persistence in the mean of prey species.

The key method used in our paper is motivated by Mao et al. (see e.g., [18, 19]), Vasilova (see e.g., [21]) and Bahar and Mao (see e.g., [3]). The significance of this paper is mainly:

(1) Gilpin-Ayala system is more suitable for the real conditions than Lotka-Volterra system, but more complicated;

(2) with adding jump type stochastic perturbations in equation, the system is more in line with the actual situation but more difficult to study.

\section{Global positive solutions}

As the $i$-th state $x_{i}(t)$ of $(1.1)$ denotes the size of the $i$-th species in the system, it should be nonnegative. In order to guarantee that SDEs have a unique global solution for any given initial value, the coefficients of the equation are generally required to satisfy both the linear growth condition and the local Lipschitz condition (see e.g., $[9,20])$. However, the coefficients of $(1.1)$ do not satisfy the linear growth condition, though they are locally Lipschitz continuous. So the solution of (1.1) may explode in a finite time. The following theorem proves that this solution is positive and global under certain conditions.

Theorem 2.1. Let Assumption 1.1 hold. Assume further there exists a constant $\mathrm{K}_{1}>0$ such that for any $\mathrm{t} \geqslant 0$,

$$
\sum_{i=1}^{d} \int_{\mathbb{Y}}\left[c_{i}(t, u)-\ln \left(1+c_{i}(t, u)\right)\right] \lambda(d u) \leqslant k_{1} .
$$

Then for any initial value $\mathrm{x}_{0} \in \mathrm{R}_{+}^{\mathrm{d}},(1.1)$ has a unique global solution $\mathrm{x}(\mathrm{t}) \in \mathrm{R}_{+}^{\mathrm{d}}$ for all $\mathrm{t} \geqslant 0$ almost surely.

Proof. Since the coefficients of the equations are locally Lipschitz continuous, for any given initial value $x_{0} \in R_{+}^{d}$ there is a unique local solution $x(t)$ on $t \in[0, \rho)$, where $\rho$ is the explosion time. To show this 
solution is global, we need to show that $\rho=\infty$ a.s.. Let $k_{0}$ be sufficiently large for every component of $x_{0}$ lying within the interval $\left[1 / k_{0}, k_{0}\right]$. For each integer $k \geqslant k_{0}$, define the stopping time

$$
\tau_{k}=\inf \left\{t \in[0, \rho) \mid x_{i}(t) \notin(1 / k, k) \text { for some } i=1, \cdots, d\right\},
$$

where throughout this paper we set $\inf \emptyset=\infty$. Clearly, $\tau_{k}$ is increasing as $k \rightarrow \infty$. Set $\tau_{\infty}=\lim _{k \rightarrow \infty} \tau_{k}$, whence $\tau_{\infty} \leqslant \rho$ a.s.. If we can show that $\tau_{\infty}=\infty$ a.s., then $\rho=\infty$ a.s. and $x(t) \in R_{+}^{d}$ a.s. for all $t \geqslant 0$. In other words, to complete the proof all we need to show is that $\tau_{\infty}=\infty$ a.s.. For if this statement is false, then there is a pair of constants $T \geqslant 0$ and $\epsilon \in(0,1)$ such that

$$
\mathbb{P}\left\{\tau_{\infty} \leqslant \mathrm{T}\right\}>\epsilon .
$$

Hence there is an integer $k_{1} \geqslant k_{0}$ such that

$$
\mathbb{P}\left\{\tau_{k} \leqslant T\right\} \geqslant \epsilon, \quad \forall k \geqslant k_{1} .
$$

Define a $C^{2}$-function $V: R_{+}^{d} \rightarrow R_{+}$by

$$
V(x)=\sum_{i=1}^{d}\left(x_{i}^{\gamma}-1-\gamma \ln x_{i}\right), \quad \gamma>0 .
$$

If $x(t) \in R_{+}^{d}$, Itô's formula shows that

$$
\begin{aligned}
\mathrm{dV}(x(t))= & F(x(t)) d t+\gamma \sum_{i=1}^{d} \sigma_{i}(t)\left(x_{i}^{\gamma}-1\right) d w(t) \\
& +\sum_{i=1}^{d} \int_{\mathbb{Y}}\left[x_{i}^{\gamma}\left(\left(1+c_{i}(t, u)^{\gamma}-1\right)-\gamma \ln \left(1+c_{i}(t, u)\right)\right) \widetilde{N}(d t, d u),\right.
\end{aligned}
$$

where for simplicity, we have omitted $t^{-}$in $x\left(t^{-}\right)$, and

$$
\begin{aligned}
F(x(t))= & \gamma\left[-\sum_{i=1}^{m} r_{i}(t)+\sum_{i=m+1}^{d} r_{i}(t)+\sum_{i=1}^{m} r_{i}(t) x_{i}^{\gamma}-\sum_{i=m+1}^{d} r_{i}(t) x_{i}^{\gamma}+\sum_{D_{2}} a_{i j}(t) x_{j}^{\alpha_{i j}}\right. \\
& \left.-\sum_{D_{1}} a_{i j}(t) x_{j}^{\alpha_{i j}}+\sum_{D_{1}} a_{i j}(t) x_{i}^{\gamma} x_{j}^{\alpha_{i j}}-\sum_{D_{2}} a_{i j}(t) x_{i}^{\gamma} x_{j}^{\alpha_{i j}}+\frac{1}{2} \sum_{i=1}^{d} \sigma_{i}^{2}(t)+\frac{\gamma-1}{2} \sum_{i=1}^{d} \sigma_{i}^{2}(t) x_{i}^{\gamma}\right] \\
& +\int_{\mathbb{Y}} \sum_{i=1}^{d} x_{i}^{\gamma}\left[\left(1+c_{i}(t, u)\right)^{\gamma}-1-\gamma c_{i}(t, u)\right] \lambda(d u)+\gamma \int_{\mathbb{Y}} \sum_{i=1}^{d}\left[c_{i}(t, u)-\ln \left(1+c_{i}(t, u)\right)\right] \lambda(d u),
\end{aligned}
$$

where $D_{2}=\{(i, j): 1 \leqslant i \leqslant m, 1 \leqslant j \leqslant d$, or $m+1 \leqslant i, j \leqslant d\}$. Under Assumption 1.1 and condition (2.1), since $c_{i}(t, u)$ are bounded functions, for $\gamma>1$, there exists a constant $K_{2}>0$ such that

$$
\begin{aligned}
F(x(t)) \leqslant & \gamma\left[-m\left(\hat{r}_{i}^{l}\right)+(d-m)\left(\check{r}_{i}^{u}\right)+\left(\check{r}_{i}^{u}\right) \sum_{i=1}^{m} x_{i}^{\gamma}+\left(\check{a}_{i i}^{u}\right) \sum_{i=1}^{d} x_{i}^{\alpha_{i i}}+\left(\check{a}_{i j}^{u}\right) \sum_{D_{1}} x_{i}^{\gamma} x_{j}^{\alpha_{i j}}\right. \\
& \left.-\left(\hat{a}_{i \mathfrak{i}}^{l}\right) \sum_{i=1}^{d} x_{i}^{\gamma+\alpha_{i i}}+\frac{d}{2}\left(\check{\sigma}_{i}^{u}\right)^{2}+\frac{\gamma-1}{2}\left(\check{\sigma}_{i}^{u}\right)^{2} \sum_{i=1}^{d} x_{i}^{\gamma}\right] \\
& +\sum_{i=1}^{d} \int_{\mathbb{Y}}\left[\left(1+c_{i}(t, u)\right)^{\gamma}-1-\gamma c_{i}(t, u)\right] \lambda(d u) x_{i}^{\gamma}+\gamma \sum_{i=1}^{d} \int_{\mathbb{Y}}\left[c_{i}(t, u)-\ln \left(1+c_{i}(t, u)\right)\right] \lambda(d u) \\
\leqslant & \left(K_{1}+K_{2}\right) \gamma,
\end{aligned}
$$


if $\gamma \leqslant 1$, using inequality $x^{\gamma} \leqslant 1+\gamma(x-1), x \geqslant 0,1 \geqslant \gamma \geqslant 0$, the above inequality can be obtained similarly.

We therefore obtain

$$
\begin{aligned}
\int_{0}^{\tau_{k} \wedge T} d V(x(t)) \leqslant & \int_{0}^{\tau_{k} \wedge T}\left(K_{1}+K_{2}\right) \gamma d t+\gamma \int_{0}^{\tau_{k} \wedge T} \sum_{i=1}^{d} \sigma_{i}(t)\left(x_{i}^{\gamma}-1\right) d w(t) \\
& +\int_{0}^{\tau_{k} \wedge T} \int_{\mathbb{Y}} \sum_{i=1}^{d}\left[x_{i}^{\gamma}\left(\left(1+c_{i}(t, u)\right)^{\gamma}-1\right)-\gamma \ln \left(1+c_{i}(t, u)\right)\right] \widetilde{N}(d t, d u) .
\end{aligned}
$$

Taking expectations, yields

$$
\mathbb{E V}\left(x\left(\tau_{\mathrm{k}} \wedge \mathrm{T}\right)\right) \leqslant \mathrm{V}\left(\mathrm{x}_{0}\right)+\left(\mathrm{K}_{1}+\mathrm{K}_{2}\right) \gamma \mathbb{E}\left(\tau_{\mathrm{k}} \wedge \mathrm{T}\right) \leqslant \mathrm{V}\left(\mathrm{x}_{0}\right)+\left(\mathrm{K}_{1}+\mathrm{K}_{2}\right) \gamma \mathrm{T}
$$

Set $\Omega_{k}=\left\{\tau_{k} \leqslant T\right\}$ for $k \geqslant k_{1}$ and by (2.2), $\mathbb{P}\left(\Omega_{k}\right) \geqslant \epsilon$. Note that for every $\omega \in \Omega_{k}$, there is some $i$ such that $x_{i}\left(\tau_{k}, \omega\right)$ equals either $k$ or $1 / k$, therefore

$$
\mathrm{V}\left(x\left(\tau_{k}, \omega\right)\right) \geqslant\left[k^{\gamma}-1-\gamma \ln (k)\right] \wedge\left[(1 / k)^{\gamma}-1-\gamma \ln (1 / k)\right]
$$

It then follows from (2.3) that

$$
\mathrm{V}\left(\mathrm{x}_{0}\right)+\left(\mathrm{K}_{1}+\mathrm{K}_{2}\right) \gamma \mathrm{T} \geqslant \mathbb{E}\left(\mathrm{I}_{\Omega_{\mathrm{k}}} \mathrm{V}\left(x\left(\tau_{\mathrm{k}}, \omega\right)\right)\right) \geqslant \epsilon\left(\left[\mathrm{k}^{\gamma}-1-\gamma \ln (\mathrm{k})\right] \wedge\left[(1 / \mathrm{k})^{\gamma}-1-\gamma \ln (1 / \mathrm{k})\right]\right) .
$$

Letting $k \rightarrow \infty$ leads to the contradiction

$$
\infty>\mathrm{V}\left(\mathrm{x}_{0}\right)+\left(\mathrm{K}_{1}+\mathrm{K}_{2}\right) \gamma \mathrm{T}=\infty,
$$

so we must have $\tau_{\infty}=\infty$ a.s. The proof is complete.

It is easy to see from this theorem that, with probability $1,(1.1)$ will not explode in a finite time.

\section{Ultimate boundedness and moment estimation}

In the previous section, we see that (1.1) has a unique global solution $x(t) \in R_{+}^{d}$ for any $t \geqslant 0$ almost surely. In this section, we discuss how the solution varies in $R_{+}^{d}$. Firstly, we discuss the ultimate boundedness.

Theorem 3.1. Suppose Assumption 1.1 holds. Let $p>0$, then the solution of (1.1) has the property

$$
\limsup _{t \rightarrow \infty} \mathbb{E}|x(t)|^{p} \leqslant \bar{K}(p),
$$

where $\overline{\mathrm{K}}(\mathrm{p})$ is a positive constant.

Proof. Define a Lyapunov function

$$
\mathrm{V}(\mathrm{x}):=\sum_{i=1}^{\mathrm{d}} x_{i}^{\mathrm{p}}, \quad x \in \mathrm{R}_{+}^{\mathrm{d}} .
$$

Applying Itô's formula yields

$$
\begin{aligned}
d\left(e^{t} V(x(t))\right)= & e^{t}[V(x(t))+\mathcal{L} V(x(t))] d t+\sum_{i=1}^{d} p e^{t} x_{i}^{p} \sigma_{i}(t, u) d w(t) \\
& +e^{t} \sum_{i=1}^{d} \int_{\mathbb{Y}} x_{i}^{p}\left[\left(1+c_{i}(t, u)\right)^{p}-1\right] \widetilde{N}(d t, d u),
\end{aligned}
$$


where we omit $t^{-}$in $x\left(t^{-}\right)$and

$$
\begin{aligned}
V(x(t))+\mathcal{L} V(x(t))= & p\left\{\frac{1}{p} \sum_{i=1}^{d} x_{i}^{p}+\sum_{i=1}^{m} r_{i}(t) x_{i}^{p}-\sum_{i=m+1}^{d} r_{i}(t) x_{i}^{p}-\sum_{i=1}^{m} \sum_{j=1}^{d} a_{i j}(t) x_{i}^{p} x_{j}^{\alpha_{i j}}\right. \\
& \left.+\sum_{i=m+1}^{d} \sum_{j=1}^{m} a_{i j}(t) x_{i}^{p} x_{j}^{\alpha_{i j}}-\sum_{i=m+1}^{d} \sum_{j=m+1}^{d} a_{i j}(t) x_{i}^{p} x_{j}^{\alpha_{i j}}+\frac{p-1}{2} \sum_{i=1}^{d} \sigma_{i}^{2}(t) x_{i}^{p}\right\} \\
& +\sum_{i=1}^{d} \int_{\mathbb{Y}}\left[\left(1+c_{i}(t, u)\right)^{p}-1-p c_{i}(t, u)\right] \lambda(d u) x_{i}^{p} .
\end{aligned}
$$

Therefore

$$
\mathbb{E}\left(e^{\mathrm{t}} \mathrm{V}(x(\mathrm{t}))\right)=\mathrm{V}\left(\mathrm{x}_{0}\right)+\mathbb{E} \int_{0}^{\mathrm{t}} e^{\mathrm{s}}[\mathrm{V}(\mathrm{x}(\mathrm{s}))+\mathcal{L} \mathrm{V}(\mathrm{x}(\mathrm{s}))] \mathrm{ds}
$$

For $p>1$, using Assumption 1.1, due to functions $c_{\mathfrak{i}}(t, u)$ are bounded, we can deduce that there exists a constant $K(p)>0$ such that

$$
\begin{aligned}
V(x(t))+\mathcal{L} V(x(t)) \leqslant & p\left\{\frac{1}{p} \sum_{i=1}^{d} x_{i}^{p}+\left(\check{r}_{i}^{u}\right) \sum_{i=1}^{m} x_{i}^{p}-\left(\hat{r}_{i}^{l}\right) \sum_{i=m+1}^{d} x_{i}^{p}+\left(\check{a}_{i j}^{u}\right) \sum_{D_{1}} x_{i}^{p} x_{j}^{\alpha_{i j}}-\left(\hat{a}_{i j}^{l}\right) \sum_{i=1}^{d} x_{i}^{p+\alpha_{i i}}\right. \\
& \left.+\frac{p-1}{2}\left(\check{\sigma}_{i}^{u}\right)^{2} \sum_{i=1}^{d} x_{i}^{p}\right\}+\sum_{i=1}^{d} \int_{\mathbb{Y}}\left[\left(1+c_{i}(t, u)\right)^{p}-1-p c_{i}(t, u)\right] \lambda(d u) x_{i}^{p} \\
\leqslant & K(p) .
\end{aligned}
$$

Hence

$$
\mathbb{E}\left(e^{t} V(x(t))\right) \leqslant V\left(x_{0}\right)+\int_{0}^{t} K(p) e^{s} d s=V\left(x_{0}\right)+K(p)\left(e^{t}-1\right)
$$

Using the inequality $|x|^{p} \leqslant d^{\frac{p}{2}} \sum_{i=1}^{d} x_{i}^{p}$ results in

$$
\limsup _{t \rightarrow \infty} \mathbb{E}|x(t)|^{p} \leqslant d^{\frac{p}{2}} \limsup _{t \rightarrow \infty} \mathbb{E}\left(\sum_{i=1}^{d} x_{i}^{p}(t)\right) \leqslant d^{\frac{p}{2}} K(p)=: \bar{K}(p) .
$$

For any $p \in(0,1]$, according to the inequality $x^{r} \leqslant 1+r(x-1), x>0,0 \leqslant r \leqslant 1$, we have

$$
\int_{\mathbb{Y}}\left[\left(1+c_{\mathfrak{i}}(t, u)\right)^{p}-1-p c_{\mathfrak{i}}(t, u)\right] \lambda(d u) \leqslant 0 .
$$

Consequently

$$
V(x(t))+\mathcal{L} V(x(t)) \leqslant p\left\{\frac{1}{p} \sum_{i=1}^{d} x_{i}^{p}+\left(\check{r}_{i}^{u}\right) \sum_{i=1}^{m} x_{i}^{p}-\left(\hat{r}_{i}^{l}\right) \sum_{i=m+1}^{d} x_{i}^{p}+\left(\check{a}_{i j}^{u}\right) \sum_{D_{1}} x_{i}^{p} x_{j}^{\alpha_{i j}}-\left(\hat{a}_{i i}^{l}\right) \sum_{i=1}^{d} x_{i}^{p+\alpha_{i i}}\right\},
$$

which has an upper bound by Assumption 1.1, and similarly to the proof of the first part the assertion (3.1) is obtained.

Definition 3.2 ([12]). The solution of (1.1) is said to be stochastically ultimately bounded, if for any $\epsilon \in(0,1)$, there is a constant $H:=H(\epsilon)$ such that for any $x_{0} \in R_{+}^{d}$,

$$
\limsup _{t \rightarrow \infty} \mathbb{P}\{|x(t)|>H\}<\epsilon .
$$


As an application of Theorem 3.1, together with the Chebyshev inequality, we have the following corollary:

Corollary 3.3. If Assumption 1.1 holds, then the solution of (1.1) is stochastically ultimately bounded.

Since (1.1) is not explicitly solvable, the study of asymptotic moment behavior is essential. In the following theorem, we prove that the time average of the p-th moment of the solution of (1.1) is asymptotically bounded.

Theorem 3.4. Assume all conditions of Theorem 2.1 hold. For $\mathrm{p}>0$, there is a positive constant $\mathrm{K}$ such that for any initial value $\mathrm{x}_{0} \in \mathrm{R}_{+}^{\mathrm{d}}$, the solution of (1.1) has the property

$$
\limsup _{t \rightarrow \infty} \frac{1}{t} \mathbb{E} \int_{0}^{t} \sum_{i=1}^{d} x_{i}^{p}(s) d s \leqslant K .
$$

Proof. From the proof of Theorem 2.1, we have obtained

$$
\begin{aligned}
\mathrm{dV}(x(t))= & F(x(t)) d t+\gamma \sum_{i=1}^{\mathrm{d}} \sigma_{\mathfrak{i}}(t)\left(x_{i}^{\gamma}-1\right) d w(t) \\
& +\sum_{i=1}^{\mathrm{d}} \int_{\mathbb{Y}}\left[x_{i}^{\gamma}\left(\left(1+c_{i}(t, u)^{\gamma}-1\right)-\gamma \ln \left(1+c_{i}(t, u)\right)\right] \widetilde{N}(d t, d u),\right.
\end{aligned}
$$

where $F(x(t))$ is the same function defined as before. Set

$$
F_{1}(x(t))=F(x(t))+\sum_{i=1}^{d} x_{i}^{p} .
$$

Then if $p<\gamma+\left(\hat{\alpha}_{i i}\right)$, under Assumption 1.1 and condition (2.1), we can deduce that there exists a constant $\mathrm{K}_{\gamma}$ such that

so it follows from (3.3),

$$
\mathrm{F}_{1}(x(\mathrm{t})) \leqslant \mathrm{K}_{\gamma}
$$

$$
\begin{aligned}
\mathrm{dV}(x(t)) \leqslant & {\left[\mathrm{K}_{\gamma}-\sum_{i=1}^{\mathrm{d}} x_{i}^{p_{i}}\right] d t+\gamma \sum_{i=1}^{\mathrm{d}} \sigma_{i}(t)\left(x_{i}^{\gamma}-1\right) \mathrm{d} w(t) } \\
& +\sum_{i=1}^{\mathrm{d}} \int_{\mathbb{Y}}\left[x_{i}^{\gamma}\left(\left(1+c_{i}(t, u)^{\gamma}-1\right)-\gamma \ln \left(1+c_{i}(t, u)\right)\right] \widetilde{N}(d t, d u) .\right.
\end{aligned}
$$

Taking expectations results in

$$
\mathbb{E V}\left(x\left(\tau_{k} \wedge t\right) \leqslant V\left(x_{0}\right)+K_{\gamma} \mathbb{E}\left(\tau_{k} \wedge t\right)-\mathbb{E} \int_{0}^{\tau_{k} \wedge t} \sum_{i=1}^{d} x_{i}^{p}(s) d s,\right.
$$

which implies

$$
\mathbb{E} \int_{0}^{\tau_{k} \wedge t} \sum_{i=1}^{d} x_{i}^{p}(s) d s \leqslant V\left(x_{0}\right)+K_{\gamma} t
$$

Letting $k \rightarrow \infty$ yields

$$
\limsup _{t \rightarrow \infty} \frac{1}{t} \mathbb{E} \int_{0}^{t} \sum_{i=1}^{d} x_{i}^{p}(s) d s \leqslant K_{\gamma} .
$$

Therefore, for any given $p>0$, we can choose $\gamma$ satisfying $\gamma+\left(\hat{\alpha}_{i i}\right)>p$ such that assertion (3.2) holds. This completes the proof. 


\section{Pathwise estimation}

The theorems in this section consider some limit inequalities for the growth rates of the population size of species which show how the solution of (1.1) varies in $R_{+}^{d}$.

Assumption 4.1. For any $t \geqslant 0, i=1, \cdots, d$, there exists a constant $c>0$ such that

$$
\int_{\mathbb{Y}}\left(\ln \left(1+c_{i}(t, u)\right)\right)^{2} \lambda(d u)<c .
$$

Theorem 4.2. Assume all conditions of Theorem 2.1 hold. Under Assumption 4.1, then there exists a positive constant $\mathrm{K}$, for any initial value $\mathrm{x}_{0} \in \mathrm{R}_{+}^{\mathrm{d}}$, the solution of (1.1) has the property

$$
\limsup _{t \rightarrow \infty} \frac{\ln \prod_{i=1}^{d} x_{i}(t)}{t} \leqslant K \text { a.s. . }
$$

Proof. We apply Itô's formula to $\ln x_{\mathfrak{i}}(\mathrm{t}), i=1, \cdots, \mathrm{m}$ and get

$$
\begin{aligned}
& d\left(\ln x_{i}(t)\right)=\left[r_{i}(t)-\sum_{j=1}^{d} a_{i j}(t) x_{j}^{\alpha_{i j}}-\frac{1}{2} \sigma_{i}^{2}(t)+\int_{\mathbb{Y}}\left(\ln \left(1+c_{i}(t, u)\right)-c_{i}(t, u)\right) \lambda(d u)\right] d t \\
& +\sigma_{\mathfrak{i}}(\mathrm{t}) \mathrm{d} w(\mathrm{t})+\int_{\mathbb{Y}} \ln \left(1+\mathrm{c}_{\mathfrak{i}}(\mathrm{t}, \mathrm{u})\right) \tilde{\mathrm{N}}(\mathrm{dt}, \mathrm{du}),
\end{aligned}
$$

which implies that

$$
\begin{aligned}
\ln x_{\mathfrak{i}}(t)= & \ln x_{\mathfrak{i}}(0)+M_{\mathfrak{i}}(\mathrm{t})+\widetilde{M}_{\mathfrak{i}}(\mathrm{t})+\int_{0}^{t}\left[r_{\mathfrak{i}}(\mathrm{s})-\sum_{j=1}^{\mathrm{d}} a_{\mathfrak{i j}}(s) x_{j}^{\alpha_{i j}}(s)-\frac{1}{2} \sigma_{\mathfrak{i}}^{2}(s)\right. \\
& \left.+\int_{\mathbb{Y}}\left(\ln \left(1+c_{\mathfrak{i}}(s, u)\right)-c_{\mathfrak{i}}(s, u)\right) \lambda(\mathrm{du})\right] \mathrm{ds},
\end{aligned}
$$

where

$$
M_{\mathfrak{i}}(\mathrm{t})=\int_{0}^{t} \sigma_{\mathfrak{i}}(\mathrm{s}) \mathrm{d} w(\mathrm{~s}), \quad \widetilde{M}_{\mathfrak{i}}(\mathrm{t})=\int_{0}^{\mathrm{t}} \int_{\mathbb{Y}} \ln \left(1+\mathrm{c}_{\mathfrak{i}}(\mathrm{s}, \mathrm{u})\right) \widetilde{\mathrm{N}}(\mathrm{ds}, \mathrm{d} \mathrm{u}),
$$

are real-valued local martingales vanishing at $t=0$ under Assumption 4.1.

Then, for $i=m+1, \cdots, d$, we again apply Itô's formula to $\ln x_{i}(t)$ and integrating from 0 to $t$ yields

$$
\begin{aligned}
\ln x_{i}(t)= & \ln x_{i}(0)+M_{i}(t)+\widetilde{M}_{i}(t) \\
& +\int_{0}^{t}\left[-r_{i}(s)+\sum_{j=1}^{m} a_{i j}(s) x_{j}^{\alpha_{i j}}(s)-\sum_{j=m+1}^{d} a_{i j}(s) x_{j}^{\alpha_{i j}}(s)-\frac{1}{2} \sigma_{i}^{2}(s)\right. \\
& \left.+\int_{\mathbb{Y}}\left(\ln \left(1+c_{i}(s, u)\right)-c_{i}(s, u)\right) \lambda(d u)\right] d s,
\end{aligned}
$$

where $M_{i}(t)$ and $\widetilde{M}_{i}(t)$ are the same real-valued local martingales mentioned above.

In the light of exponential martingale inequality with jumps [2], fix $\epsilon \in(0,1)$ arbitrarily and let $\theta>1$, for every integer $n \geqslant 1$, we obtain

$$
\begin{aligned}
\mathbb{P}\left\{\operatorname { s u p } _ { 0 \leqslant t \leqslant n } \left[\int_{0}^{t} \sigma_{i}(s) d w(s)-\frac{\epsilon}{2} \int_{0}^{t} \sigma_{i}^{2}(s) d s+\int_{0}^{t} \int_{\mathbb{Y}} \ln \left(1+c_{i}(s, u)\right) \tilde{N}(d s, d u)\right.\right. \\
\left.\left.\quad-\frac{1}{\epsilon} \int_{0}^{t} \int_{\mathbb{Y}}\left(e^{\epsilon \ln \left(1+c_{i}(s, u)\right)}-1-\epsilon \ln \left(1+c_{\mathfrak{i}}(s, u)\right)\right) \lambda(d u) d s\right] \geqslant \frac{\theta \ln n}{\epsilon}\right\}<\frac{1}{n^{\theta}} .
\end{aligned}
$$


Since the series $\sum_{n=1}^{\infty} \frac{1}{n^{\theta}}$ converges, we can deduce from the Borel-Cantella lemma [20] that there exists an $\Omega_{i} \subseteq \Omega$ with $\mathbb{P}\left(\Omega_{i}\right)=1$ such that for any $\omega \in \Omega_{i}$, an integer $n_{i}=n_{i}(\omega)$ can be found such that

$$
\begin{aligned}
\int_{0}^{t} \sigma_{i}(s) d w(s)+\int_{0}^{t} \int_{\mathbb{Y}} \ln \left(1+c_{i}(s, u)\right) \widetilde{N}(d s, d u) & \leqslant \frac{\theta \ln n}{\epsilon}+\frac{\epsilon}{2} \int_{0}^{t} \sigma_{i}^{2}(s) d s \\
& +\int_{0}^{t} \int_{\mathbb{Y}}\left[c_{i}(s, u)-\ln \left(1+c_{i}(s, u)\right)\right] \lambda(d u) d s,
\end{aligned}
$$

whenever $n \geqslant n_{i}, 0 \leqslant t \leqslant n$. Moreover, according to the equality $\ln x \leqslant x-1$ when $x>0$,

$$
\int_{\mathbb{Y}}\left(\ln \left(1+c_{\mathfrak{i}}(t, u)-c_{\mathfrak{i}}(t, u)\right)\right) \lambda(d u) \leqslant 0
$$

Thus for $i=1, \cdots, m$ it follows from (4.1)

$$
\begin{aligned}
\ln x_{\mathfrak{i}}(t) \leqslant & \ln x_{\mathfrak{i}}(0)+\frac{\theta \ln n}{\epsilon}+\int_{0}^{t} \int_{\mathbb{Y}}\left(c_{i}(s, u)-\ln \left(1+c_{i}(s, u)\right)\right) \lambda(d u) d s \\
& +\int_{0}^{t}\left[r_{i}(s)-\sum_{j=1}^{d} a_{i j}(s) x_{j}^{\alpha_{i j}}(s)-\frac{1-\epsilon}{2} \sigma_{i}^{2}(s)\right] d s
\end{aligned}
$$

and for $i=m+1, \cdots, d$ from (4.2) it follows

$$
\begin{aligned}
\ln x_{i}(t) \leqslant & \ln x_{i}(0)+\frac{\theta \ln n}{\epsilon}+\int_{0}^{t} \int_{\mathbb{Y}}\left(c_{i}(s, u)-\ln \left(1+c_{i}(s, u)\right)\right) \lambda(d u) d s \\
& +\int_{0}^{t}\left[-r_{i}(s)+\sum_{j=1}^{m} a_{i j}(s) x_{j}^{\alpha_{i j}}(s)-\sum_{j=m+1}^{d} a_{i j}(s) x_{j}^{\alpha_{i j}}(s)-\frac{1-\epsilon}{2} \sigma_{i}^{2}(s)\right] d s,
\end{aligned}
$$

for $0 \leqslant t \leqslant n_{i}$ and $n \geqslant n_{i}$ whenever $\omega \in \Omega_{i}$. Denote $\Omega_{0}=\bigcap_{i=1}^{d} \Omega_{i}$. It is easy to see $\mathbb{P}\left(\Omega_{0}\right)=1$. Moreover, for any $\omega \in \Omega_{0}$, let $n_{0}(\omega)=\max \left\{n_{i}(\omega): 1 \leqslant i \leqslant d\right\}$. Then, for any $\omega \in \Omega_{0}$, from (4.3) and (4.4) we get

$$
\sum_{i=1}^{d} \ln x_{i}(t) \leqslant \sum_{i=1}^{d} \ln x_{i}(0)+\frac{\theta d \ln n}{\epsilon}+\int_{0}^{t} \int_{\mathbb{Y}} \sum_{i=1}^{d}\left(c_{i}(s, u)-\ln \left(1+c_{i}(s, u)\right)\right) \lambda(d u) d s+\int_{0}^{t} F(x(s)) d s,
$$

where

$$
\begin{aligned}
F(x(s))= & \sum_{i=1}^{m} r_{i}(s)-\sum_{i=m+1}^{d} r_{i}(s)-\sum_{i=1}^{m} \sum_{j=1}^{d} a_{i j}(s) x_{j}^{\alpha_{i j}}(s)+\sum_{i=m+1}^{d} \sum_{j=1}^{m} a_{i j}(s) x_{j}^{\alpha_{i j}}(s) \\
& -\sum_{i=m+1}^{d} \sum_{j=m+1}^{d} a_{i j}(s) x_{j}^{\alpha_{i j}}(s)-\frac{1-\epsilon}{2} \sum_{i=1}^{d} \sigma_{i}^{2}(s),
\end{aligned}
$$

for all $0 \leqslant t \leqslant n$ and $n \geqslant n_{0}$.

According to Assumption 1.1, there exists a constant $K_{2}>0$ such that

$$
F(x(s)) \leqslant m\left(\check{r}_{i}^{u}\right)-(d-m)\left(\hat{r}_{i}^{l}\right)+\left(\check{a}_{i j}^{u}\right) \sum_{D_{1}} \chi_{j}^{\alpha_{i j}}(s)-\left(\hat{a}_{i i}^{l}\right) \sum_{i=1}^{d} \chi_{i}^{\alpha_{i i}}(s)-\frac{1-\epsilon}{2} d\left(\hat{\sigma}_{i}^{l}\right)^{2} \leqslant K_{2} .
$$

Combining with (2.1) yields

$$
\sum_{i=1}^{d} \ln x_{i}(t) \leqslant \sum_{i=1}^{d} \ln x_{i}(0)+\frac{\theta d \ln n}{\epsilon}+\left(K_{1}+K_{2}\right) t
$$

for $0 \leqslant \mathrm{t} \leqslant \mathrm{n}, \mathrm{n} \geqslant \mathrm{n}_{0}$. 
Consequently, for any $\omega \in \Omega_{0}$, if $n-1 \leqslant t \leqslant n$ and $n \geqslant n_{0}+1$, we obtain

$$
\limsup _{t \rightarrow \infty} \frac{\ln \prod_{i=1}^{\mathrm{d}} x_{i}(t)}{t} \leqslant \limsup _{n \rightarrow \infty}\left[\frac{\ln \prod_{i=1}^{d} x_{i}(0)}{n-1}+\frac{\theta d \ln n}{\epsilon(n-1)}+\left(K_{1}+K_{2}\right)\right]=: K .
$$

This completes the proof.

Theorem 4.3. Under Assumptions 1.1 and 4.1, if in addition the following condition holds: for any $\mathrm{t} \geqslant 0$, and $\mathrm{i}=1, \cdots, \mathrm{d}$,

$$
\sup _{t \geqslant 0} \int_{0}^{t} \int_{\mathbb{Y}} e^{\lambda(s-t)}\left[c_{\mathfrak{i}}(s, u)-\ln \left(1+c_{\mathfrak{i}}(s, u)\right)\right] \lambda(d u) d s<\infty,
$$

then, for any initial value $\mathrm{x}_{0} \in \mathrm{R}_{+}^{\mathrm{d}}$

$$
\limsup _{t \rightarrow \infty} \frac{\ln \left(\prod_{i=1}^{\mathrm{d}} x_{i}(t)\right)}{\ln t} \leqslant d \text { a.s. . }
$$

Proof. Let $\lambda>0$. For $i=1, \cdots, m$, we apply Itô's formula to $e^{\lambda t} \ln x_{i}(t)$ and get

$$
\begin{aligned}
e^{\lambda t} \ln x_{i}(t)= & \ln x_{i}(0)+\int_{0}^{t} e^{\lambda s} \sigma_{i}(s) d w(s)+\int_{0}^{t} \int_{\mathbb{Y}} e^{\lambda s} \ln \left(1+c_{i}(s, u)\right) \widetilde{N}(d s, d u) \\
& +\int_{0}^{t} e^{\lambda s}\left[\lambda \ln x_{i}(s)+r_{i}(s)-\sum_{j=1}^{d} a_{i j}(s) x_{j}^{\alpha_{i j}}(s)-\frac{1}{2} \sigma_{i}^{2}(s)\right] d s \\
& +\int_{0}^{t} \int_{\mathbb{Y}} e^{\lambda s}\left(\ln \left(1+c_{i}(s, u)\right)-c_{i}(s, u)\right) \lambda(d u) d s .
\end{aligned}
$$

Similarly, for $i=m+1, \cdots, d$, we get

$$
\begin{aligned}
e^{\lambda t} \ln x_{i}(t)= & \ln x_{i}(0)+\int_{0}^{t} e^{\lambda s} \sigma_{i}(s) d w(s)+\int_{0}^{t} \int_{\mathbb{Y}} e^{\lambda s} \ln \left(1+c_{i}(s, u)\right) \widetilde{N}(d s, d u) \\
& +\int_{0}^{t} e^{\lambda s}\left[\lambda \ln x_{i}(s)-r_{i}(s)+\sum_{j=1}^{m} a_{i j}(s) x_{j}^{\alpha_{i j}}(s)-\sum_{j=m+1}^{d} a_{i j}(s) x_{j}^{\alpha_{i j}}(s)-\frac{1}{2} \sigma_{i}^{2}(s)\right] d s \\
& +\int_{0}^{t} \int_{\mathbb{Y}} e^{\lambda s}\left(\ln \left(1+c_{i}(s, u)\right)-c_{i}(s, u)\right) \lambda(d u) d s .
\end{aligned}
$$

For any $\epsilon \in(0,1)$ arbitrarily, $\theta>1$ and every integer $n \geqslant 1$, under Assumption 4.1 , using the exponential martingale inequality with jumps yields

$$
\begin{aligned}
& \mathbb{P}\left\{\operatorname { s u p } _ { 0 \leqslant t \leqslant n } \left[\int_{0}^{t} e^{\lambda s} \sigma_{i}(s) d w(s)-\frac{\epsilon}{2} e^{-\lambda n} \int_{0}^{t} e^{2 \lambda s} \sigma_{i}^{2}(s) d s+\int_{0}^{t} \int_{\mathbb{Y}} e^{\lambda s} \ln \left(1+c_{i}(s, u)\right) \widetilde{N}(d s, d u)\right.\right. \\
& \left.\left.-\frac{1}{\epsilon e^{-\lambda n}} \int_{0}^{t} \int_{\mathbb{Y}}\left(e^{\epsilon e^{-\lambda n} e^{\lambda s} \ln \left(1+c_{\mathfrak{i}}(s, u)\right)}-1-\epsilon e^{-\lambda n} e^{\lambda s} \ln \left(1+c_{\mathfrak{i}}(s, u)\right)\right) \lambda(d u) d s\right] \geqslant \frac{\theta e^{\lambda n} \ln n}{\epsilon}\right\}<\frac{1}{n^{\theta}} .
\end{aligned}
$$

Since the series $\sum_{n=1}^{\infty} \frac{1}{n^{\theta}}<\infty$, from the Borel-Cantella lemma we obtain there is an $\Omega_{i} \subseteq \Omega$ with $\mathbb{P}\left(\Omega_{\mathfrak{i}}\right)=1$ such that for any $\omega \in \Omega_{\mathfrak{i}}$, an integer $n_{\mathfrak{i}}=n_{\mathfrak{i}}(\omega)$ can be found such that

$$
\begin{aligned}
\int_{0}^{t} e^{\lambda s} \sigma_{\mathfrak{i}}(s) d w(s)+\int_{0}^{t} \int_{\mathbb{Y}} e^{\lambda s} \ln \left(1+c_{\mathfrak{i}}(s, u)\right) \widetilde{N}(d s, d u) \leqslant & \frac{\theta e^{\lambda n} \ln n}{\epsilon}+\frac{\epsilon}{2} e^{-\lambda n} \int_{0}^{t} e^{2 \lambda s} \sigma_{i}^{2}(s) d s \\
& +\int_{0}^{t} \int_{\mathbb{Y}} e^{\lambda s}\left[c_{\mathfrak{i}}(s, u)-\ln \left(1+c_{i}(s, u)\right)\right] \lambda(d u) d s,
\end{aligned}
$$


whenever $n \geqslant n_{i}, 0 \leqslant t \leqslant n$. In addition,

$$
\int_{\mathbb{Y}}\left(\ln \left(1+c_{\mathfrak{i}}(t, u)-c_{\mathfrak{i}}(t, u)\right)\right) \lambda(d u) \leqslant 0 .
$$

Now let $\Omega_{0}=\bigcap_{i=1}^{d} \Omega_{i}$. Clearly $\mathbb{P}\left(\Omega_{0}\right)=1$. Moreover, for any $\omega \in \Omega_{0}$, let

$$
n_{0}(\omega)=\max \left\{n_{i}(\omega): 1 \leqslant i \leqslant d\right\} .
$$

Then, for any $\omega \in \Omega_{0}$, by using inequalities (4.8) and (4.9), it follows from (4.6), (4.7)

$$
\begin{aligned}
e^{\lambda t} \sum_{i=1}^{d} \ln x_{i}(t) \leqslant & \sum_{i=1}^{d} \ln x_{i}(0)+\frac{\theta d e^{\lambda n} \ln n}{\epsilon} \\
& +\int_{0}^{t} \int_{\mathbb{Y}} e^{\lambda s} \sum_{i=1}^{d}\left(c_{i}(s, u)-\ln \left(1+c_{i}(s, u)\right)\right) \lambda(d u) d s+\int_{0}^{t} e^{\lambda s} \widetilde{F}(x(s)) d s,
\end{aligned}
$$

for all $0 \leqslant t \leqslant n$ and $n \geqslant n_{0}(\omega)$, where

$$
\begin{aligned}
\widetilde{F}(x(s))= & \lambda \sum_{i=1}^{d} \ln x_{i}(s)+\sum_{i=1}^{m} r_{i}(s)-\sum_{i=m+1}^{d} r_{i}(s)-\sum_{i=1}^{m} \sum_{j=1}^{d} a_{i j}(s) x_{j}^{\alpha_{i j}}(s)+\sum_{i=m+1}^{d} \sum_{j=1}^{m} a_{i j}(s) x_{j}^{\alpha_{i j}}(s) \\
& -\sum_{i=m+1}^{d} \sum_{j=m+1}^{d} a_{i j}(s) x_{j}^{\alpha_{i j}}(s)-\frac{1-\epsilon e^{\lambda s-\lambda n}}{2} \sum_{i=1}^{d} \sigma_{i}^{2}(s) \\
\leqslant & \lambda \sum_{i=1}^{d} \ln x_{i}(s)+m\left(\check{r}_{i}^{u}\right)-(d-m)\left(\hat{r}_{i}^{l}\right)+\left(\check{a}_{i j}^{u}\right) \sum_{D_{1}} x_{j}^{\alpha_{i j}}(s)-\left(\hat{a}_{i j}^{l}\right) \sum_{i=1}^{d} x_{i}^{\alpha_{i i}}(s)-\frac{1-\epsilon e^{\lambda s-\lambda n}}{2} d\left(\hat{\sigma}_{i}^{l}\right)^{2},
\end{aligned}
$$

which has an upper bound under Assumption 1.1, say $\widetilde{K}$. So for any $\omega \in \Omega_{0}$, if $(n-1) \leqslant t \leqslant n$ and $n \geqslant n_{0}+1$, we obtain

$$
\begin{aligned}
\frac{\ln \prod_{i=1}^{\mathrm{d}} x_{i}(t)}{\ln t} \leqslant & \frac{\ln \prod_{i=1}^{\mathrm{d}} x_{i}(0)}{e^{\lambda t} \ln t}+\frac{\theta d e^{\lambda n} \ln n}{\epsilon e^{\lambda(n-1)} \ln (n-1)}+\frac{1}{\ln t} \int_{0}^{t} \widetilde{K} e^{\lambda(s-t)} d s \\
& +\frac{1}{\ln t} \int_{0}^{t} \int_{\mathbb{Y}} e^{\lambda(s-t)}\left(c_{i}(s, u)-\ln \left(1+c_{i}(s, u)\right)\right) \lambda(d u) d s .
\end{aligned}
$$

Supposing that $\mathrm{n} \uparrow \infty$, using (4.5) leads to

$$
\limsup _{t \rightarrow \infty} \frac{\ln \prod_{i=1}^{d} x_{i}(t)}{\ln t} \leqslant \frac{\theta d e^{\lambda}}{\epsilon} \text { a.s., }
$$

and the conclusion follows on setting $\lambda \downarrow 0, \epsilon \uparrow 1$ and $\theta \downarrow 1$.

Remark 4.4. Under the conditions of Theorem 4.2, from the above proof, it is easy to see that for $i=$ $1, \cdots, d, \limsup _{t \rightarrow \infty} \frac{\ln x_{i}(t)}{\ln t} \leqslant 1$ a.s.. Noting the $\operatorname{limit}_{\lim } \rightarrow \infty \frac{\ln t}{t}=0$, we have

$$
\limsup _{t \rightarrow \infty} \frac{\ln x_{i}(t)}{t} \leqslant 0 \text { a.s. . }
$$

\section{The survival analysis for (1.1)}

Definition 5.1 ([16]).

(1) Stochastic population $x_{i}(t)$ is said to be extinct with probability 1 , if for every initial value $x_{0} \in R_{+}^{d}$, the solution $x_{i}(t), t \geqslant 0$ has the property

$$
\lim _{t \rightarrow \infty} x_{i}(t)=0 \text { a.s. . }
$$


(2) Stochastic population $x_{i}(t)$ is said to be weakly persistent in the mean, if

$$
\limsup _{t \rightarrow \infty} \frac{1}{t} \int_{0}^{t} x_{i}(s) d s>0 \text { a.s. . }
$$

Theorem 5.2. Under Assumptions 1.1 and 4.1.

(i) If for $\mathrm{t} \geqslant 0$,

$$
\limsup _{t \rightarrow \infty} \frac{1}{t} \int_{0}^{t} \beta_{i}(s) d s<0, \quad i=1, \cdots, m,
$$

where

$$
\beta_{i}(t):=r_{i}(t)-\frac{1}{2} \sigma_{i}^{2}(t)-\int_{\mathbb{Y}}\left(c_{i}(t, u)-\ln \left(1+c_{i}(t, u)\right)\right) \lambda(d u),
$$

then all prey species and predator species are extinctive a.s. .

(ii) If $\lim \sup _{\mathrm{t} \rightarrow \infty} \frac{1}{\mathrm{t}} \int_{0}^{\mathrm{t}} \beta_{\mathrm{i}}(\mathrm{s}) \mathrm{d} s>0, i=1, \cdots, \mathrm{m}$ and condition (4.5) holds, then for any initial value $\mathrm{x}_{0} \in \mathrm{R}_{+}^{\mathrm{d}}$, there exist constants $b_{i}>0, i=1, \cdots, m$ such that

$$
\limsup _{t \rightarrow \infty} \frac{1}{t} \int_{0}^{t} x_{i}(s) d s>b_{i}, i=1, \cdots, m, \quad \text { a.s. . }
$$

Proof. (i) Applying Itô's formula to $\ln x_{i}(t), i=1, \cdots, m$ gives

$$
\frac{\ln \left(x_{i}(t) / x_{i}(0)\right)}{t} \leqslant \frac{1}{t} \int_{0}^{t} \beta_{i}(s) d s+\frac{M_{i}(t)}{t}+\frac{\widetilde{M}_{i}(t)}{t}
$$

where $M_{i}(t)=\int_{0}^{t} \sigma_{i}(s) d w(s)$, and $\widetilde{M}_{i}(t)=\int_{0}^{t} \int_{\mathbb{Y}} \ln \left(1+c_{i}(s, u)\right) \widetilde{N}(d s, d u)$. Noting that

$$
\left\langle M_{i}\right\rangle(t)=\int_{0}^{t} \sigma_{i}^{2}(s) d s \leqslant\left(\check{\sigma}_{i}^{u}\right)^{2} t
$$

and

$$
\left\langle\widetilde{M}_{i}\right\rangle(t)=\int_{0}^{t} \int_{\mathbb{Y}}\left[\ln \left(1+c_{i}(s, u)\right)\right]^{2} \lambda(d u) d s \leqslant c t,
$$

where $\langle\cdot\rangle(t)$ is Meyer's angle bracket process.

From strong law of large numbers [20], we obtain

$$
\lim _{t \rightarrow \infty} \frac{M_{i}(t)}{t}=0, \text { a.s. and } \lim _{t \rightarrow \infty} \frac{\widetilde{M}_{i}(t)}{t}=0 \text { a.s. . }
$$

Combining with (5.1), yields

$$
\limsup _{t \rightarrow \infty} \frac{\ln x_{i}(t)}{t} \leqslant \limsup _{t \rightarrow \infty} \frac{1}{t} \int_{0}^{t} \beta_{i}(s) d s<0 .
$$

So for $i=1, \cdots, m$,

$$
\lim _{t \rightarrow \infty} x_{i}(t)=0 \text { a.s. . }
$$

That is, all prey species become extinct.

For $i=m+1, \cdots, d$, applying Itô's formula to $\ln x_{i}(t)$ and integrating from 0 to $t$ yields

$$
\begin{aligned}
\frac{\ln x_{i}(t)}{t} \leqslant & \frac{\ln x_{i}(0)}{t}+\frac{M_{i}(t)}{t}+\frac{\widetilde{M}_{i}(t)}{t} \\
& +\frac{1}{t} \int_{0}^{t}\left[-r_{i}(s)+\left(\check{a}_{i j}^{\mathfrak{u}}\right) \sum_{j=1}^{m} x_{j}^{\alpha_{i j}}(s)-\frac{1}{2} \sigma_{i}^{2}(s)-\int_{\mathbb{Y}}\left(c_{i}(s, u)-\ln \left(1+c_{i}(s, u)\right)\right) \lambda(d u)\right] d s .
\end{aligned}
$$


By (5.3), (5.4)

$$
\limsup _{t \rightarrow \infty} \frac{\ln x_{i}(t)}{t}<0
$$

so for $i=m+1, \cdots, d$,

$$
\lim _{t \rightarrow \infty} x_{i}(t)=0 \text { a.s., }
$$

i.e., all predator species become extinct.

(ii) If (5.2) is false, then for any fixed $\epsilon>0$, there exists a solution $\widetilde{x}(t)$ with initial value $\widetilde{x}_{0} \in R_{+}^{d}$ such that

$$
\mathbb{P}\left\{\limsup _{t \rightarrow \infty} \frac{1}{t} \int_{0}^{t} \widetilde{x}_{i}(s) d s<\epsilon\right\}>0, \quad i=1, \cdots, m .
$$

Letting $\epsilon$ be sufficiently small to satisfy

$$
\begin{aligned}
& \limsup _{t \rightarrow \infty} \frac{1}{t} \int_{0}^{t} \beta_{i}(s) d s-\left(\check{a}_{i j}^{u}\right) \sum_{j=1}^{m} \epsilon^{\alpha_{i j}}>0, \quad i=1, \cdots, m, \\
& \limsup _{t \rightarrow \infty} \frac{1}{t} \int_{0}^{t} \eta_{i}(s) d s+\left(\check{a}_{i j}^{u}\right) \sum_{j=1}^{m} \epsilon^{\alpha_{i j}}<0, \quad i=m+1, \cdots, d,
\end{aligned}
$$

where $\eta_{i}(t):=-r_{i}(t)-\frac{1}{2} \sigma_{i}^{2}(t)-\int_{\mathbb{Y}}\left(c_{i}(t, u)-\ln \left(1+c_{i}(t, u)\right)\right) \lambda(d u)$.

For $i=m+1, \cdots, d$, from (4.2), (5.3) and (5.5), we have

$$
\limsup _{t \rightarrow \infty} \frac{\ln \widetilde{x}_{i}(t)}{t} \leqslant \limsup _{t \rightarrow \infty} \frac{1}{t} \int_{0}^{t} \eta_{i}(s) d s+\left(\check{a}_{i j}^{u}\right) \sum_{j=1}^{m} \epsilon^{\alpha_{i j}}<0,
$$

therefore

$$
\lim _{t \rightarrow \infty} \widetilde{x}_{i}(t)=0, \quad i=m+1, \cdots, d .
$$

On the other hand, for $i=1, \cdots, m$, from (4.1), we obtain

$$
\frac{\ln \left(\widetilde{x}_{i}(t) / x_{i}(0)\right)}{t} \geqslant \frac{1}{t} \int_{0}^{t} \beta_{i}(s) d s-\left(\check{a}_{i j}^{u}\right) \frac{1}{t} \int_{0}^{t} \sum_{j=1}^{m} \widetilde{x}_{j}^{\alpha_{i j}}(s)-\left(\check{a}_{i j}^{u}\right) \frac{1}{t} \int_{0}^{t} \sum_{j=m+1}^{d} \widetilde{x}_{j}^{\alpha_{i j}}(s)+\frac{M_{i}(t)}{t}+\frac{\widetilde{M}_{i}(t)}{t} .
$$

Substituting (5.3), (5.5) into (5.6) results in

$$
\limsup _{t \rightarrow \infty} \frac{\ln \widetilde{x}_{i}(t)}{t} \geqslant \limsup _{t \rightarrow \infty} \frac{1}{t} \int_{0}^{t} \beta_{i}(s) d s-\left(\check{a}_{i j}^{u}\right) \sum_{j=1}^{m} \epsilon^{\alpha_{i j}}>0, \quad i=1, \cdots, m .
$$

In other words, we have shown that

$$
\mathbb{P}\left\{\limsup _{t \rightarrow \infty} \frac{\ln \tilde{x}_{i}(t)}{t}>0\right\}>0, \quad i=1, \cdots, m,
$$

which contradicts (4.10). This completes the proof.

Remark 5.3. Making use of inequality $x-1-\ln x \geqslant 0, x>0$, yields

$$
\beta_{\mathfrak{i}}(s):=r_{\mathfrak{i}}(s)-\frac{1}{2} \sigma_{i}^{2}(s)-\int_{\mathbb{Y}}\left(c_{\mathfrak{i}}(s, u)-\ln \left(1+c_{\mathfrak{i}}(s, u)\right)\right) \lambda(d u) \leqslant r_{i}(s)-\frac{1}{2} \sigma_{i}^{2}(s) .
$$

Thus Theorem 5.2 reveals that jumps can make the population extinct.

Remark 5.4. From Theorem 5.2, it is easy to see that prey species are weakly persistent in the mean. 


\section{Conclusions}

This paper is concerned with a general non-autonomous Gilpin-Ayala $m$ prey and $(d-m)$ predator species model with jumps. Gilpin-Ayala model is a highly nonlinear system, so it is difficult to discuss this model. In this paper, we adopt the analysis of Lyapunov functions which has been used by many authors to discuss the asymptotic behavior of the solution of our model. We show that the model with jumps admits a unique global positive solution, and investigate stochastic ultimate boundedness, asymptotic moment estimation and pathwise estimation. From the survival analysis of this model, we obtain that jumps can make the population extinct.

\section{Acknowledgment}

This work was supported by the National Natural Science of China (No.11501571) and the Fundamental Research Funds for the Central Universities (No.16CX02011A). I would like to express my sincere thanks to the editors and reviewers for their very helpful comments and suggestions, which greatly improved the quality of this paper.

\section{References}

[1] A. S. Ackleh, D. F. Marshall, H. E. Heatherly, Extinction in a generalized Lotka-Volterra predator-prey model, J. Appl. Math. Stochastic Anal., 13 (2000), 287-297. 1

[2] D. Applebaum, Lévy processes and stochastic calculus, Second edition, Cambridge Studies in Advanced Mathematics, Cambridge University Press, Cambridge, (2009). 4

[3] A. Bahar, X.-R. Mao, Stochastic delay population dynamics, Int. J. Pure Appl. Math., 11 (2004), 377-399. 1

[4] J.-H. Bao, X.-R. Mao, G. Yin, C.-G. Yuan, Competitive Lotka-Volterra population dynamics with jumps, Nonlinear Anal., 74 (2011), 6601-6616. 1

[5] J.-H. Bao, C.-G. Yuan, Stochastic population dynamics driven by Lévy noise, J. Math. Anal. Appl., 391 (2012), $363-375$. 1

[6] F.-D. Chen, C.-L. Shi, Global attractivity in an almost periodic multi-species nonlinear ecological model, Appl. Math. Comput., 180 (2006), 376-392. 1

[7] M. Fan, K. Wang, Global periodic solutions of a generalized n-species Gilpin-Ayala competition model, Comput. Math. Appl., 40 (2000), 1141-1151. 1

[8] M. E. Gilpin, F. J. Ayala, Global models of growth and competition, Proc. Natl. Acad. Sci. U.S.A., 70 (1973), $3590-3593$. 1

[9] R. Z. Hasminski1̆, Stochastic stability of differential equations, Translated from the Russian by D. Louvish, Monographs and Textbooks on Mechanics of Solids and Fluids: Mechanics and Analysis, Sijthoff \& Noordhoff, Alphen aan den Rijn-Germantown, Md., (1980). 2

[10] A. Korobeinikov, G. C. Wake, Global properties of the three-dimensional predator-prey Lotka-Volterra systems, J. Appl. Math. Decis. Sci., 3 (1999), 155-162. 1

[11] C. R. Li, S. J. Lu, Qualitative analysis of $\mathrm{N}$-species prey-competition systems with nonlinear relations and periodic coefficients, (Chinese) Gaoxiao Yingyong Shuxue Xuebao Ser. A, 12 (1997), 147-156. 1

[12] X.-Y. Li, X.-R. Mao, Population dynamical behavior of non-autonomous Lotka-Volterra competitive system with random perturbation, Discrete Contin. Dyn. Syst., 24 (2009), 523-545. 1, 3.2

[13] B.-S. Lian, S.-G. Hu, Stochastic delay Gilpin-Ayala competition models, Stoch. Dyn., 6 (2006), 561-576. 1

[14] B.-S. Lian, S.-G. Hu, Asymptotic behaviour of the stochastic Gilpin-Ayala competition models, J. Math. Anal. Appl., 339 (2008), 419-428. 1

[15] X.-Y. Liao, S.-F. Zhou, Y.-M. Chen, On permanence and global stability in a general Gilpin-Ayala competition predatorprey discrete system, Appl. Math. Comput., 190 (2007), 500-509. 1

[16] M. Liu, K. Wang, Persistence and extinction of a stochastic single-specie model under regime switching in a polluted environment, J. Theoret. Biol., 264 (2010), 934-944. 5.1

[17] A. J. Lotka, Elements of mathematical biology, (formerly published under the title Elements of Physical Biology), Dover Publications, Inc., New York, (1958). 1

[18] X.-R. Mao, G. Marion, E. Renshaw, Environmental Brownian noise suppresses explosions in population dynamics, Stochastic Process. Appl., 97 (2002), 95-110. 1, 1

[19] X.-R. Mao, S. Sabanis, E. Renshaw, Asymptotic behaviour of the stochastic Lotka-Volterra model, J. Math. Anal. Appl., 287 (2003), 141-156. 1, 1

[20] X.-R. Mao, C.-G. Yuan, Stochastic differential equations with Markovian switching, Imperial College Press, London, (2006). 2, 4, 5 
[21] M. Vasilova, Asymptotic behavior of a stochastic Gilpin-Ayala predator-prey system with time-dependent delay, Math. Comput. Modelling, 57 (2013), 764-781. 1, 1, 1

[22] M. Vasilova, M. Jovanović, Dynamics of Gilpin-Ayala competition model with random perturbation, Filomat, 24 (2010), 101-113. 1

[23] M. Vasilova, M. Jovanović, Stochastic Gilpin-Ayala competition model with infinite delay, Appl. Math. Comput., 217 (2011), 4944-4959. 1

[24] V. Volterra, Variazioni e fluttuazioni del numero d'individui in specie d'animali conviventi, Mem. Acad. Lincei, 2 (1926), 31-113. 1 